\title{
Psoriasis and alcohol
}

This article was published in the following Dove Press journal:

Psoriasis: Targets and Therapy

\author{
Caroline Svanström ${ }^{1,2}$ \\ Sol-Britt Lonne-Rahm ' \\ Klas Nordlind ${ }^{2}$ \\ 'Department of Dermatology, \\ Mälarsjukhuset, Eskilstuna, Sweden; \\ ${ }^{2}$ Department of Medicine, Solna, \\ Dermatology and Venereology Unit, \\ Karolinska Institutet, Stockholm, Sweden
}

\begin{abstract}
Psoriasis is a chronic inflammatory skin disease that may be triggered or worsened by several factors, including alcohol. A higher than average alcohol consumption is common among individuals with psoriasis. Neurobiological signaling affected by alcohol intake includes a range of neurotransmitters, such as the dopaminergic, serotonergic, and tachykinergic systems, involved in reward and drug-seeking. These neurotransmitters may also have an impact on the inflammatory processes per se in psoriasis. Future therapy may, therefore, be targeted at neurotransmitter networks involved with both alcohol intake and the inflammatory processes.
\end{abstract}

Keywords: psoriasis, alcohol, pruritus, neurotransmitters

\section{Introduction}

Psoriasis is a chronic inflammatory skin disease affecting $2-5 \%$ of the population in Western countries. The disease is characterized by increased cell proliferation in the basal epidermal layer and disturbed differentiation in the apical part of the epidermis. Pathoetiological mechanisms include both innate and acquired immune systems. ${ }^{1}$

There is a complex interplay between genetic and environmental factors linked to the severity and triggering of psoriasis. ${ }^{2,3}$ Such triggering/worsening factors include damage to the skin and streptococcal infection. Studies have also shown that psychological stress, due to emergency crises and family stress, may contribute to the onset of psoriasis. Other factors that can worsen psoriasis are drugs, such as beta-blockers, lithium, and interferon- $\alpha$. Smoking may also have a negative effect on the disease. ${ }^{4}$ Recently Zink et $\mathrm{al}^{5}$ reported various forms of addiction in psoriasis, with alcohol dependence being a substantial one.

The present review discusses the role of alcohol (ethanol) in psoriasis.

\section{General effects of alcohol}

Alcohol by itself can be harmful, but there is an important role of its oxidative metabolite acetaldehyde, in disruption of the intestinal barrier, associated with alcohol consumption. ${ }^{6}$ This disruption acts synergistically with acetaldehyde. Alcohol and its metabolite acetone may promote keratinocyte proliferation in vitro and may thus enhance the permeability of the skin, disrupting its barrier function. $^{7}$

Alcohol also has an effect on the metabolism of lipids, such as triglycerides and cholesterol, and may thus also affect the lipid composition of the skin barrier. ${ }^{8}$ Alcohol has been shown to induce rapid peroxidation of lipids in cerebral vascular smooth muscle cells in rats, which may be a triggering pathway for
Correspondence: Caroline Svanström Department of Medicine, Solna,

Dermatology and Venereology Unit, Karolinska Institutet, Stockholm SE I7I 76, Sweden

Tel +46702307268

Email svanstrom.caroline@gmail.com 
proinflammatory events in alcohol-induced brain vascular injury. ${ }^{9}$ It may also activate microglia and neurons in rats. $^{10}$

Alcohol may be both an initiating and aggravating factor in inflammation. It can affect both innate and adaptive immunity. Previous studies have suggested mechanisms such as increased proinflammatory cytokines, eg, increased production of tumor necrosis factor $-\alpha$ (TNF- $\alpha$ ) from peripheral blood monocytes and macrophages, and increased lymphocyte proliferation and activation, caused by alcohol. ${ }^{11}$ Alcohol-induced liver injury in mice resulted in an impairment of skin barrier function, possibly mediated via plasma TNF- $\alpha{ }^{12}$ Alcohol may also have an impact on the number and function of murine epidermal $\mathrm{T}$ cells. $^{13}$ The methylation changes in $\mathrm{CD}^{+} \mathrm{T}$ cells are greater in patients with psoriasis than in healthy control subjects. ${ }^{14}$ Alcohol may have an impact on migration of murine dermal dendritic cells to draining lymph nodes after sensitization. ${ }^{15}$ In female mice, alcohol caused a reduced dendritic function, compared to male mice. ${ }^{16}$

Mast cells are believed to play a role in the pathogenesis of psoriasis, and alcohol may affect the number of mast cells as well as their degranulation. ${ }^{17-19}$

The skin and central nervous system share a common origin through the ectoderm.

Alcohol affects the brain in several ways. Like other drugs, it stimulates the brain's reward center via several neurotransmitter systems, depending on the phase of alcohol dependency of the individual. ${ }^{20}$

\section{Alcohol and psoriasis}

Patients with psoriasis consume more alcohol than the general population. ${ }^{21}$ Alcohol may affect psoriasis through several mechanisms, such as increased susceptibility to infections, stimulation of lymphocyte and keratinocyte proliferation, and production of proinflammatory cytokines. $^{11,22}$

Only a limited number of studies have investigated the relationship between the severity of psoriasis and alcohol use. In these, mostly retrospective, studies, a positive correlation between alcohol intake and the extent of psoriasis has, in general, been reported for women. An association between total body surface area and alcohol use was found in a case-control study on women in- and out patients with mixed subtypes of psoriasis. ${ }^{23}$ A multicenter study of 1203 inpatients with different subtypes of psoriasis demonstrated a correlation between the psoriasis area severity index (PASI) score and level of alcohol consumption, but only for women. ${ }^{24}$ The level of alcohol use was estimated based on medical record data, and the majority of patients were social drinkers according to self-report. In a limited cross-sectional study, we have shown a correlation in women between alcohol consumption, measured by phosphatidylethanol (PEth) and Lifetime Drinking History (LDH) interview, and extent of psoriasis. ${ }^{25}$ However, an earlier case-control study showed alcohol to be a risk factor for psoriasis in young and middle-aged men. ${ }^{26}$

There are few prospective studies on the role of alcohol in psoriasis. In the Nurses' Health Study (NHS II), intake of non light beer in a cohort of US nurses was associated with an increased risk of developing psoriasis. ${ }^{27}$ In 150 outpatients enrolled in a 3-month observational study, therapeutic outcome, as measured by achieving 75\% PASI recovery, with conventional psoriasis therapy, was hampered due to obesity, female sex, tobacco or smoking habits, and alcohol habits. ${ }^{28}$ In a Pakistani study on outpatients with psoriasis, PASI score was significantly associated with drinking alcohol. ${ }^{29}$ In the Department of Dermatology, Karolinska University Hospital, Solna, we have performed a small prospective study on 22 patients with low-moderate intake of alcohol and mild-moderate extent of psoriasis, using Christmas and New Year as the exposure periods, when alcohol intake is expected to increase. ${ }^{30}$ Although alcohol intake increased, there was only a marginal increase in the extent of psoriasis.

Alcohol consumption has not only been associated with a more severe and a higher incidence of psoriasis, but also with a distinctive nature and distribution of the disease. Patients with psoriasis and alcohol overconsumption tend to have more severe inflammation, with minimal scale, typically distributed in the face, groin, and flexures, or typically hyperkeratotic lesions with a predominant acral distribution. ${ }^{31}$

Alcohol intake may induce pruritus by lowering its threshold and thus has been suggested to increase pruritus in psoriasis. However, several studies have failed to show such an association. ${ }^{25,32,33}$

A study of 95 patients with plaque psoriasis examined their alcohol intake and psychological stress and found that $17-30 \%$ of patients were rated as having alcohol problems. Thirteen percent and $18 \%$ of patients with psoriasis were classified with ongoing or previous alcohol problems, respectively, and there was a link between increased alcohol intake and increased anxiety and depression. In addition, the patients who considered that they had 
alcohol problems had more trouble with anxiety and depression, and also more psoriasis. ${ }^{34}$

People with psoriasis have an increased risk of premature mortality. The reason for this is not fully understood, but is likely to be multifactorial. The most common comorbidities for patients with psoriasis are hyperlipidemia, hypertension, depression, diabetes mellitus type 2, and obesity. ${ }^{35}$ A Finnish cohort admitted to inpatient treatment with psoriasis as the principal diagnosis showed an excess mortality caused by alcohol. Among men, these causes included alcohol-related psychosis, liver disease, and alcohol dependence, and, in women, liver disease. ${ }^{36}$ A population-based cohort study investigated whether people with psoriasis had a higher risk of alcohol-related mortality and found that patients with psoriasis had a $60 \%$ greater risk of dying due to alcohol-related causes compared to others of the same age and sex in the general population. This indicated that alcohol may be a great contributor to the premature mortality gap. The predominant causes of alcohol-related deaths were alcoholic liver disease, fibrosis and cirrhosis of the liver, and psychological and behavioral disorders due to alcohol. ${ }^{37}$ There is an increased proportion of liver disease, both alcoholic and non alcoholic, in psoriatic patients, and the prevalence of psoriasis in patients with alcoholic liver disease is higher than the predicted value in the normal population. ${ }^{31,38}$ In this respect, TNF- $\alpha$, a key cytokine in psoriasis pathogenesis, may have a role.

\section{Therapeutic neurotransmitter targets}

The current therapy for psoriasis per se includes topical desquamating drugs, potent topical glucocorticoids, topical calcipotriol, and, for severe disease, systemic treatments, such as UV light, methotrexate, and biologicals, such as anti-TNF- $\alpha$ agents. A problem with regard to classical systemic treatments, such as biologicals, is that, due to inhibition of cells that destroy invading microorganisms, alcohol intake may result in increased susceptibility to infections. $^{22}$

It should also be noted that alcohol use may be associated with worse adherence to treatment of their skin disease. In addition, alcohol consumption may be used by patients with psoriasis to cope with their debilitating skin disease. ${ }^{38}$

It may be attractive to use a neuro endocrine approach for patients with psoriasis who present with overconsumption of alcohol.
Targets for treatment of alcohol dependence per se may focus on the neuronal circuits of reward, addiction, and satisfaction, in addition to the biochemical pathways of alcohol metabolism. ${ }^{20}$ These include dopaminergic, serotonergic, tachykinergic and acetylcholine (ACh), opioid, gamma-aminobutyric acid (GABA), and glutamatergic-mediated transmissions. Pharmacologic drugs in such systems used for treatment of alcohol dependence are: dopamine (DA) 2 receptor (R) and serotonin (5-HT)2R antagonists (eg, olanzapine), selective serotonin reuptake inhibitors (SSRIs) (eg, sertraline), 5-HT3R antagonist (ondansetron), and naltrexone. As regards the tachykinergic system, recently neurokinin (NK)-1R antagonism has been implicated in modulating alcohol consumption and reward. ${ }^{39,40}$

Regarding the inflammatory process in psoriasis per se, we and/or others have found receptors for biogenic amines, such as 5-HT, ${ }^{41,42}$ tachykinins, including the NK-1R, ${ }^{43}$ and ACh receptors, ${ }^{44}$ in psoriasis. Expression of such receptors has been found in the basal and/or differentiating zones of the epidermis and on dermal inflammatory cells, while dendritic inflammatory cells in the epidermis and dermis show expression of SERT. ${ }^{45}$ A cohort study by Thorslund et al $^{46}$ found that patients with psoriasis who had undergone SSRI treatment were less often switched to systemic treatment. Interestingly, brainstem 5-HT transporter availability has been shown to be correlated with circulating TNF- $\alpha{ }^{47} 5$ HT2R activation may suppress TNF- $\alpha$-induced inflammation, ${ }^{48}$ and polymorphism of the 5-HT2AR gene is associated with late-onset psoriasis in a Thai population. ${ }^{49}$

A relationship between DA and the pathophysiology of psoriasis has been known since the 1970s. Thus, levodopa resulted in the resolution of inverse psoriasis when being used for Parkinson's disease, possibly due to an increase in cyclic AMP. ${ }^{50}$ Moreover, bromocriptine, a strong D2R agonist, has been used in patients with psoriasis with different results on the clinical outcome. ${ }^{51-53}$

The anti depressive drug bupropion, a noradrenaline and DA reuptake inhibitor, has been reported to result in a reduction in psoriasis-affected skin surface. ${ }^{54}$

N-methyl-D-aspartate is a glutamate receptor abnormally expressed in psoriasis. Disturbed expression of subunits of such receptors has been reported in the basal and apical part of the epidermis. ${ }^{55,56}$ GABA and $\mathrm{GABA}_{\mathrm{A}} \mathrm{R}$ expression has previously been found mainly in inflammatory cells in psoriasis. ${ }^{57}$

Future therapies for alcohol and psoriasis might thus be targeted at neurotransmitter networks involved with both alcohol intake and the inflammatory processes. 


\section{Conclusion}

More research is needed in order to determine whether alcohol can induce or worsen psoriasis. Studies should examine whether there is a threshold to alcohol intake at which the disease worsens or debuts, and whether the type of alcohol plays a part and, if so, to what extent.

It is difficult to interpret study results, because patients with psoriasis may smoke and suffer from metabolic syndrome, ie, there are several confounders. Furthermore, the measure of alcohol intake differs in different studies, and it is, therefore, difficult to compare results and draw a valid conclusion.

There are difficulties in recruiting patients with psoriasis to participate in studies concerning the correlation of their disease with alcohol intake. Stigmatization concerning both alcohol and psoriasis may make it difficult to recruit a sufficient number of patients. In this respect, register studies or studies in which patients may answer inquiries and rate their ongoing intake of alcohol and the extent of their psoriasis anonymously would be of importance. Also, the effect of ceasing alcohol intake on the extent of the disease should be studied.

Animal experiments are warranted; however, such experiments are at present limited by the lack of a suitable animal model for psoriasis.

In addition, more prospective studies, including during seasonal periods/festivals when patients consume a higher than normal amount of different types of alcohol, may be of substantial value.

\section{Disclosure}

The authors report no conflicts of interest in this work.

\section{References}

1. Ogawa E, Sato Y, Minagawa A, Okuyama R. Pathogenesis of psoriasis and development of treatment. J Dermatol. 2018;45(3):264-272. doi:10.1111/1346-8138.14139

2. Zhang P, Su Y, Lu Q. Epigenetics and psoriasis. J Eur Acad Dermatol Venereol. 2012;26(4):399-403. doi:10.1111/j.1468-3083.2011.04261.x

3. Zhao M, Lu Q. The aberrant epigenetic modifications in the pathogenesis of psoriasis. J Investig Dermatol Symp Proc. 2018;19(2):S81-S82. doi:10.1016/j.jisp.2018.09.007

4. Zeng J, Luo S, Huang Y, Lu Q. Critical role of environmental factors in the pathogenesis of psoriasis. J Dermatol. 2017;44(8):863-872. doi:10.1111/jde.2017.44.issue-8

5. Zink A, Herrmann M, Fischer T, et al. Addiction: an underestimated problem in psoriasis health care. $J$ Eur Acad Dermatol Venereol. 2017;31(8):1308-1315. doi:10.1111/jdv.14326

6. Elamin EE, Masclee AA, Dekker J, Jonkers DM. Ethanol metabolism and its effects on the intestinal epithelial barrier. Nutr Rev. 2013;71 (7):483-499. doi:10.1111/nure.12027

7. Farkas A, Kemény L, Széll M, Dobozy A. Ethanol and acetone stimulate the proliferation of HaCaT keratinocytes: the possible role of alcohol in exacerbating psoriasis. Arch Dermatol Res. 2003;295 (2):56-62. doi:10.1007/s00403-003-0399-2
8. Park H, Kim K. Association of alcohol consumption with lipid profile in hypertensive men. Alcohol Alcohol. 2012;47(3):282-287. doi:10.1093/alcalc/ags019

9. Altura BM, Gebrewold A, Zhang A, Altura BT. Ethanol induces rapid lipid peroxidation and activation of nuclear factor-kappa $B$ in cerebral vascular smooth muscle: relation to alcohol-induced brain injury in rats. Neurosci Lett. 2002;325(2):95-98. doi:10.1016/S03043940(02)00264-1

10. Walter TJ, Vetreno RP, Crews FT. Alcohol and stress activation of microglia and neurons: brain regional effects. Alcohol Clin Exp Res. 2017;41(12):2066-2081.

11. Farkas A, Kemény L. Psoriasis and alcohol; is cutaneous ethanol one of the missing links. Br J Dermatol. 2010;162(4):711-716. doi:10.1111/ j.1365-2133.2009.09595.x

12. Yokoyama S, Hiramoto K, Koyama M, Ooi K. Chronic liver injury in mice promotes impairment of skin barrier function via tumor necrosis factor-alpha. Cutan Ocul Toxicol. 2016;35(3):194-203. doi:10.3109/ 15569527.2015.1076433

13. Parlet CP, Waldschmidt TJ, Schlueter AJ. Chronic ethanol feeding induces subset loss and hyporesponsiveness in skin T cells. Alcohol Clin Exp Res. 2014;38(5):1356-1364. doi:10.1111/acer.2014.38.issue-5

14. Park GT, Han J, Park SG, Kim S, Kim TY. DNA methylation analysis of CD4+ T cells in patients with psoriasis. Arch Dermatol Res. 2014;306(3):259-268. doi:10.1007/s00403-013-1432-8

15. Parlet CP, Schlueter AJ. Mechanisms by which chronic ethanol feeding impairs the migratory capacity of cutaneous dendritic cells. Alcohol Clin Exp Res. 2013;37(12):2098-2107. doi:10.1111/j.15300277.2012.01883.x

16. Thompson MG, Navarro F, Chitsike L, Ramirez L, Kovacs EJ, Watkins SK. Alcohol exposure differentially effects anti-tumor immunity in females by altering dendritic cell function. Alcohol. 2016;57:1-8. doi:10.1016/j.alcohol.2016.10.007

17. Harvima I, Nilsson G, Suttle M, Naukkarinen A. Is there a role for mast cells in psoriasis? Arch Dermatol Res. 2008;300(9):461-478. doi:10.1007/s00403-007-0806-1

18. Zimatkin SM, Anichtchik OV. Alcohol-histamine interactions. Alcohol Alcohol. 1999;34(2):141-147. doi:10.1093/alcalc/34.2.141

19. Mendes LO, Amorim JP, Teixeira GR, et al. Mast cells and ethanol consumption: interactions in the prostate, epididymis and testis of UChB rats. Am J Reprod Immunol. 2011;66(3):170-178. doi:10.1111/ j.1600-0897.2010.00958.x

20. Ferraguti G, Pascale E, Lucarelli M. Alcohol addiction: a molecular biology perspective. Curr Med Chem. 2015;22(6):670-684. doi: $10.2174 / 0929867321666141229103158$

21. Brenaut E, Horreau C, Pouplard C, et al. Alcohol consumption and psoriasis: a systematic literature review. J Eur Acad Dermatol Venereol. 2013;27(Suppl 3):30-35. doi:10.1111/jdv.12164

22. Farkas A, Kemény L. Alcohol, liver, systemic inflammation and skin: a focus on patients with psoriasis. Skin Pharmacol Physiol. 2013;26 (3): $119-126$. doi: $10.1159 / 000348865$

23. Poikolainen K, Reunala T, Karvonen J. Smoking, alcohol and life events related to psoriasis among women. Br J Dermatol. 1994;130 (4):473-477. doi:10.1111/j.1365-2133.1994.tb03396.x

24. Gerdes S, Zahl VA, Weichenthal M, Mrowietz U. Smoking and alcohol intake in severely affected patients with psoriasis in Germany. Dermatology. 2010;220(1):38-43. doi:10.1159/000265557

25. Zou L, Lonne-Rahm SB, Helander A, Stokkeland K, Franck J, Nordlind $\mathrm{K}$. Alcohol intake measured by phosphatidylethanol in blood and the lifetime drinking history interview are correlated with the extent of psoriasis. Dermatology. 2015;230(4):375-380. doi:10.1159/000380818

26. Poikolainen K, Reunala T, Karvonen J, Lauharanta J, Kärkkäinen P. Alcohol intake: a risk factor for psoriasis in young and middle aged men? BMJ. 1990;300(6727):780-783. doi:10.1136/bmj.300.6727.780

27. Qureshi AA, Dominguez PL, Choi HK, Han J, Curhan G. Alcohol intake and risk of incident psoriasis in US women. Arch Dermatol. 2010;146(12):1364-1369. doi:10.1001/archdermatol.2010.156 
28. Gupta AK, Pandey SS, Pandey BL. Effectiveness of conventional drug therapy of plaque psoriasis in the context of consensus guidelines: a prospective observational study in 150 patients. Arch Dermatol. 2013;25(2):156-162.

29. Khawaja AR, Bokhari SMA, Rasheed T, et al. Disease severity, quality of life, and psychiatric morbidity in patients with psoriasis with reference to sociodemographic, lifestyle, and clinical variables: a prospective, crosssectional study from Lahore, Pakistan. Prim Care Companion CNS Disord. 2015;17(3). doi:10.4088/PCC.14m01629

30. Svanström C, Bircan E, Stokkeland K, Franck J, Nordlind K. A prospective study on the link between alcohol and psoriasis. Abstract. 47th annual ESDR meeting, September 27-30, 2017, Salzburg, Austria. J Invest Dermatol. 2017;137(Suppl 2):S204.

31. Cassano N, Vesitita M, Apruzzi D, Vena GA. Alcohol, psoriasis, liver disease, and anti-psoriasis drugs. Int J Dermatol. 2011;50(11):13231331. doi:10.1111/j.1365-4632.2011.05008.x

32. Gupta MA, Gupta AK, Kirkby S, et al. Pruritus in psoriasis. A prospective study of some psychiatric and dermatologic correlates. Arch Dermatol. 1988;124(7):1052-1057. doi:10.1001/archderm. 1988.01670070040016

33. Reich A, Szepietowski JC. Clinical aspects of itch: Psoriasis. In: Itch: Mechanisms and Treatment. Carstens E, Akiyama T, editors. Chapter 4. Boca Raton (FL): CRC Press/Taylor\&Francis; 2014: 33-46.

34. Kirby B, Richards HL, Mason DL, Fortune DG, Main CJ, Griffiths CE. Alcohol consumption and psychological distress in patients with psoriasis. Br J Dermatol. 2008;158(1):138-140.

35. Shah K, Mellars L, Changolkar A, Feldman SR. Real-world burden of comorbidities in US patients with psoriasis. $J$ Am Acad Dermatol. 2017;77(2):287-292. doi:10.1016/j.jaad.2017.03.037

36. Poikolainen K, Karvonen J, Pukkala E. Excess mortality related to alcohol and smoking among hospital-treated patients with psoriasis. Arch Dermatol. 1999;135(12):1490-1493. doi:10.1001/archderm. 135.12.1490

37. Parisi R, Webb RT, Carr MJ, et al. Alcohol-related mortality in patients with psoriasis: a population-based cohort study. JAMA Dermatol. 2017;153(12):1256-1262. doi:10.1001/jamadermatol.2017.3225

38. Tobin AM, Higgins EM, Norris S, Kirby B. Prevalence of psoriasis in patients with alcoholic liver disease. Clin Exp Dermatol. 2009;34 (6):698-701. doi:10.1111/j.1365-2230.2008.03140.x

39. Rinker JA, Mulholland PJ. Promising pharmacogenetic targets for treating alcohol use disorder: evidence from preclinical models. Pharmacogenomics. 2017;18(6):1-17. doi:10.2217/pgs-2016-0193

40. Schank JR, Heilig M. Substance P and the neurokinin-1 receptor: the new CRF. Int Rev Neurobiol. 2017;136:151-175.

41. Slominski AT, Zmijewski MA, CezarySkobowiat C, et al. Sensing the environment: regulation of local and global homeostasis by the skin neuroendocrine system. Adv Anat Embryol Cell Biol. 2012;212:v.

42. Nordlind K, Azmitia EC, Slominski A. The skin as a mirror of the soul: exploring the possible roles of serotonin. Exp Dermatol. 2008;17(4):301-311. doi:10.1111/exd.2008.17.issue-4

43. Remröd C, Lonne-Rahm S, Nordlind K. Study of substance P and its receptor neurokinin-1 in psoriasis and their relation to chronic stress and pruritus. Arch Dermatol Res. 2007;299(2):85-91. doi:10.1007/ s00403-007-0745-x
44. Radosa J, Dyck W, Goerdt S, Kurzen H. The cholinergic system in guttate psoriasis with special reference to mast cells. Exp Dermatol. 2011;20(8):677-679. doi:10.1111/j.1600-0625.2011.0 1283.x

45. Thorslund K, El-Nour H, Nordlind K. The serotonin transporter protein is expressed in psoriasis, where it may play a role in regulating apoptosis. Arch Dermatol Res. 2009;301(6):449-457. doi:10.1007/s00403-009-0933-y

46. Thorslund K, Svensson T, Nordlind K, Ekbom A, Fored CM. Use of serotonin reuptake inhibitors in patients with psoriasis is associated with a decreased need for systemic psoriasis treatment: a populationbased cohort study. J Intern Med. 2013;274(3):281-287. doi:10.1111/ joim. 12093

47. Krishnadas R, Nicol A, Sassarini J, et al. Circulating tumour necrosis factor is highly correlated with brainstem serotonin transporter availability in humans. Brain Behav Immun. 2016;51:29-38. doi:10.1016/ j.bbi.2015.08.005

48. Yu B, Becnel J, Zerfaoui M, Rohatgi R, Boulares AH, Nichols CD. Serotonin 5-hydroxytryptamine(2A) receptor activation suppresses tumor necrosis factor-alpha-induced inflammation with extraordinary potency. J Pharmacol Exp Ther. 2008;327(2):316-323. doi:10.1124/ jpet.108.139337

49. Ronpirin C, Tencomnao T, Wongpiyabovorn J. Association between the $-1438 \mathrm{~A} / \mathrm{G}$ polymorphism of the serotonin $2 \mathrm{~A}$ receptor gene and late-onset psoriasis in a Thai population. Genet Mol Res. 2010;9 (1):208-214. doi:10.4238/vol9-1gmr718

50. Rojo Suárez N, Jiménez Gallo D, Arjona Aguilera C, Espinosa Rosso $\mathrm{R}$, Linares Barrios M. Resolution of inverse psoriasis after treatment with levodopa for Parkinson's disease. Dermatol Ther. 2017;30(1): e12408. doi: $10.1111 /$ dth. 12408

51. Weber G, Neidhardt M, Frey H, Galle K, Geiger A. Treatment of psoriasis with bromocriptin. Arch Dermatol Res. 1981;271(4):437439. doi:10.1007/BF00406689

52. Guilhou JJ, Guilhou E. Bromocriptine treatment of psoriasis. Arch Dermatol Res. 1982;273(1-2):159-160. doi:10.1007/BF00509042

53. AbouKhedr NA, Eid AA. Clinical efficacy of bromocriptine and the influence of serum prolactin levels on disease severity in patients with chronic plaque-type psoriasis. Alexandria J Med. 2013;49 (4):385-389. doi:10.1016/j.ajme.2013.04.001

54. Modell JG, Boyce S, Taylor E, Katholi C. Treatment of atopic dermatitis and psoriasis vulgaris with bupropion-SR: a pilot study. Psychosom Med. 2002;64(5):835-840.

55. Fischer M, William T, Helmbold P, Wohlrab J, Marsch WCH. Expression of epidermal N-methyl-D-aspartate receptors (NMDAR1) depends on formation of the granular layer-analysis in diseases with parakeratotic cornification. Arch Dermatol Res. 2004;296(4):157-162.

56. Morhenn VB, Nelson TE, Gruol DL. The rate of wound healing is increased in psoriasis. $J$ Dermatol Sci. 2013;72(2):87-92. doi:10.1016/j.jdermsci.2013.06.001

57. Nigam R, El-Nour H, Amatya B, Nordlind K. GABA and GABA(A) receptor expression on immune cells in psoriasis: a pathophysiological role. Arch Dermatol Res. 2010;302(7):507-515. doi:10.1007/ s00403-010-1052-5

Psoriasis: Targets and Therapy

Dovepress

Publish your work in this journal

Psoriasis: Targets and Therapy is international, peer-reviewed, open access journal focusing on psoriasis, nail psoriasis, psoriatic arthritis and related conditions, identification of therapeutic targets and the

optimal use of integrated treatment interventions to achieve improved outcomes and quality of life. Visit http://www.dovepress com/testimonials.php to read real quotes from published authors.

Submit your manuscript here: http://www.dovepress.com/psoriasis-targets-and-therapy-journal 